\title{
Network Performance Management Using Realistic Abductive Reasoning Model
}

\author{
G. Prem Kumar and P. Venkataram \\ Department of Electrical Communication Engineering \\ Indian Institute of Science \\ Bangalore - 560 012, INDIA \\ (Tel: (+91) (080) 3340855; Fax: (+91) (080) 3347991 \\ e-mail : $\{$ prem, pallapa $\} @ e c e . i i s c . e r n e t . i n)$
}

\begin{abstract}
Performance degradation in communication networks can be viewed to be caused by a set of faults, called soft failures, owing to which the network resources like bandwidth can not be utilized to the expected level. An automated solution to the performance management problem involves identifying these soft failures and use/suggest suitable remedies to tune the network for better performance. Abductive reasoning model is identified as a suitable candidate for the network performance management problem. An approach to solve this problem using the realistic abductive reasoning model is proposed. The realistic abductive inference mechanism is based on the parsimonious covering theory with some new features added to the general abductive reasoning model. The network performance management knowledge is assumed to be represented in the most general form of causal chaining, namely, hyper-bipartite network. Ethernet performance management is taken up as a case study. The results obtained by the proposed approach demonstrate its effectiveness in solving the network performance management problem.
\end{abstract}

\section{Keywords}

Network Performance Management, Network Fault Diagnosis, Realistic Abductive Reasoning Model, Parsimonious Covering Theory, Ethernet Performance Management.

\section{INTRODUCTION}

Communication network management (Cassel,1989), (Sluman, 1989) is drawing a lot of attention as the networks are spreading geographically and the number of heterogeneous devices and services supported by them are increasing exponentially. Network performance management is a complex part of present day network management (Hayes, 1993). The necessity for performance management arises when the network continues to function but in a degraded fashion because of one or more of the reasons such as 
temporary congestion that causes delayed transmission, failure of higher level protocols and mischevious users (Metcafe, 1976). In this work, the performance degradation is considered as a soft failure since the network is only partially afffected but is still in operation; on the other hand, if some of the devices in the network are not functioning or if the network is not able to run, then it is considered as a hard failure.

There are some specialized problems in the network management, that have to be considered. The entire information required for management may not be available at once and there may be missing information, both of which, the management center needs to confirm with the respective managed nodes. In this paper, we present a two step approach that aids the network performance management. First step involves identification of a set of faults from the given soft failures by using Realistic Abductive Reasoning Model (Realistic_ARM) (Prem, 1994) that is modelled as a diagnostic problem solver. In the second step, the system suggests suitable remedies to tune the network for better performance.

The fundamental idea behind abductive reasoning is "reasoning to the best explanation" (Pople, 1973). Based on the given symptoms (or manifestations), initially, it uses forward chaining to anticipate all the possible causes of the symptoms (also called disorders), and then it uses backward chaining to confirm whether the explanation is supported to a required degree of confidence. Ever since parsimonious covering theory (Reggia, 1985), (Peng, 1987), (Peng, 1990) is developed for abductive reasoning with sound mathematical foundation, there has been a shift in attention from deductive reasoning to abductive reasoning. Abductive reasoning generates all the possible explanations which may require further refinement to arrive at appropriate covers (Bylander, 1991). Deductive reasoning, though generates only appropriate covers, will not generate those required covers which it would have generated in the presence of missing information. Both, abductive and deductive reasoning strategies are far from the reality. The proposed approach, which uses Realistic_ARM for solving the network performance management problem is a compromise between the two strategies and attempts to find explanations for a given set of symptoms. The knowledge used by Realistic_ARM is assumed to be represented in the most general form of causal chaining, namely, hyperbipartite network.

We briefly describe the realistic abductive reasoning model in Section 2. Section 3 discusses the network performance management problem and highlights the applicability of realistic abductive reasoning model in solving the problem. The algorithm is presented in Section 4. A case study, Ethernet performance management is discussed in Section 5. And finally, conclusion follows in Section 6 .

\section{THE REALISTIC ABDUCTIVE REASONING MODEL}

Realistic abductive reasoning model (Prem, 1994) is a modified version of the abductive reasoning model (Peng, 1990) to solve the diagnostic problems effectively in a realistic scenario. This model uses abductive inference mechanism based on the parsimonious covering theory with some new features added to the general model of diagnostic problem solving. 


\subsection{Notation}

Definition 1: The diagnostic problem, $P$, is a 4-tuple $\langle M, D, H, L\rangle$ where $M=$ $\left\{m_{1}, m_{2}, \ldots, m_{e}\right\}$ is a set of manifestations causing a set of disorders, $D=\left\{d_{1}, d_{2}, \ldots, d_{f}\right\}$ either directly or via a set of hypotheses (which could be a manifestation or a disorder), $H=\left\{h_{1}, h_{2}, \ldots, h_{r}\right\}$. And, $L=\left\{l_{i, j} \mid i \in M \cup H, j \in H \cup D\right\}$ is a set of causal links joining any two related elements in $M, H$ and $D$. In a general case, there are many causes to each of the manifestations, many effects to each of the disorders, and both causes and effects to each of the hypotheses.

Definition 2: Hyper-bipartite network is an acyclic graph, $\mathrm{G}=\langle M, D, H, L\rangle$, where $M$ is a set of manifestations (in the bottom most layer), $D$ is a set of disorders (in the top most layer) and $H$ is a set of hypotheses (in one or more intermediate layers). All elements of $M, H$, and $D$ are represented as nodes in their respective layers. And, $L$ is a set of edges joining any two related nodes in $M, H$ and $D$. Let the number of layers in the graph be $N$.

Definition 3: Layered network is an acyclic graph $G^{*}=\left\langle M, D, H^{*}, L^{*}\right\rangle$, constructed from the hyper-bipartite network $G$, where each node belonging to $M, H^{*}$ and $D$ are connected only to the nodes in its neighboring layers. The procedure to convert a hyper-bipartite network into a layered network, Build_Layered_Net, is discussed in Section 4.

Definition 4:A symptom is an observed manifestation/hypothesis/disorder.

Definition 5: A volunteered symptom is a hypothesis/disorder at layer $i(1<i \leq N)$ observed to be present.

A hypothesis/disorder covers a symptom if there is a causal pathway from the hypothesis/disorder to the symptom.

Definition 6: A cover or an explanation is a set of hypotheses/disorders that covers all the given symptoms.

In solving the diagnostic problem, $P$, where the representation is in the form of a layered network, $G^{*}, j$ th cover of layer $i(1 \leq \mathrm{i}<\mathrm{N}), c_{j}{ }^{i}=\left\{h_{1}, h_{2}, \ldots, h_{s}\right\}$, is a set of disorders at layer $(i+1)$, which covers the symptoms at layer $i$. At each layer, there may be more than one explanation for the given symptoms and they are placed in the cover set of that layer, $C_{i}=\left\{c_{1}{ }^{i}, c_{2}{ }^{i}, \ldots, c_{t}{ }^{i}\right\}$. While at the top most layer, a volunteered symptom is simply added to each cover of the cover set if it is not already present.

Definition $7:$ Intermediate cover $\left(t_{k}^{i}\right)$, of layer $i$, is a cover belonging to the the cover set $\left(T_{i}\right)$ being generated, which provides an explanation for the symptoms being explored but may or may not provide explanation for the unexplored symptoms.

Definition 8: Direct disorder, $d d \in D$, of a manifestation/hypothesis is the direct cause of the manifestation/hypothesis mapping on to the top most layer.

Definition 9: Irredundancy is the parsimonious criteria used in Realistic_ARM to refine the cover set by eliminating the redundant covers. A cover $c_{j}^{i}$ is redundant if there exists another cover $c_{k}^{i}$, which is a subset of $c_{j}^{i}$.

Definition 10 : The solution to a diagnostic problem is the set of all explanations for the given symptoms.

\subsection{The Realistic_ARM}

Inference process used in the abductive reasoning, based on parsimonious covering theory, is similar to the model of sequential hypothesis-test cycle of human diagnostic problem 
solving (Peng, 1990). The "hypothesis" part covers the given symptoms and generates parsimonious covers. The "test" part of it is the question-answering process to explore for more symptoms to discriminate the generated covers. This cycle continues, taking one symptom at a time, until all relevant questions are asked and all symptoms are processed.

The diagnostic knowledge in Realistic_ARM is represented in the form of a hyperbipartite network. In this model, all the manifestations/hypotheses have direct disorders. All the elements belonging to $M, D, H^{*}$ exist only in their respective layers. Any symptom belonging to any layer may appear at any time during the reasoning process. All the possible manifestations that could be present in a layer because of the existing manifestions through common disorders (the disorder a manifestation causes along with some other manifestations/hypotheses) are queried at once before starting the reasoning process for that layer. The advantage here is two fold : (i) all the covers will be generated with the same set of symptoms, and (ii) especially in the networking environment, queries for the presence of manifestations need a lot of time in collecting the information and it is good to present them at the earliest.

In the rest of this section, we describe the realistic abductive reasoning model approach to solve a general diagnostic problem.

Solution to the diagnostic problem where the knowledge base is represented in the form of a hyper-bipartite network is found by converting it into a layered network and solving it as a series of bipartite networks, moving upwards one layer at a time. A cover for the symptoms in layer $(i-1), c_{j}^{i-1}$, becomes symptoms for layer $i . \quad\left(C_{0}\right.$ is initialized to $\{\emptyset\}$.) In addition to these, some more symptoms that are added at layer $i$ by user input (or interactive querying) together form $j$ th symptom set at layer $i$, for which an intermediate cover set $T_{i}$ is built in the following way : at layer $i$, starting with a symptom, all its disorders get into different covers since each of them separately provide an explanation for that symptom. For the subsequent symptoms, if a cover is already providing the explanation, the cover will remain unchanged. Otherwise, for an intermediate cover, $t_{k}^{i}$, that is not providing an explanation for a symptom, $m_{l}$, append only those disorders of $m_{l}$, which are supported by prespecified number of symptoms, one at a time to form new covers and delete $t_{k}^{i}$. If no new covers are generated, then append the direct disorder of $m_{l}$ to $t_{k}^{i}$. After the covers are built to provide explanation to all the symptoms, the parsimonious criteria, namely, irredundancy is applied and a few covers are eliminated. $T_{i}$ is then appended to the cover set $C_{i}$ and reinitialized to $\{\emptyset\}$ to take up next symptom set of that layer. When all the symptom sets are explored, $C_{i}$ is made irredundant. This process repeats for all the layers till the top most layer is reached. At the top most layer, the volunteered symptoms are simply added to each cover of the cover set if they are not already present. After covering the symptoms of the top most layer, if there are any more symptoms left uncovered, the reasoning process repeats from the bottom most layer. The intention here is to cover the symptoms only at their respective layer along with the other symptoms of that layer to avoid too much of guess in generating the covers and retain the simple layered network architecture with out additional dummy nodes. For details, refer (Prem, 1994). 


\section{ADAPTATION OF REALISTIC_ARM TO SOVLE NETWORK PERFORMANCE MANAGEMENT PROBLEM}

The fact that the Realistic_ARM is a compromise between the extreme cases of abductive and deductive reasoning models is utilized to solve the network performance management problem. Since in the network scenario, there may be missing information and all the infomation required for fault identification may not be available at the time of diagnosis. If the deductive reasoning mechanism is applied to such a problem, the fault can not be identified since all the symptoms may not be present. At the same time, the abductive reasoning approach will result in too many number of unwanted explanations for a given set of symptoms and subsequently, it will be very difficult to say which is the correct explanation (a set of faults), that caused the degradation in the network performance. The realistic abductive reasoning model discussed in the previous section can be found to satisfy the requirements of the problem.

The prespecified number of symptoms required to support a given symptom before concluding a disorder (fault) is a variable. This can be set based on the incremental step in which the performance needs to be tuned. Intermediate layer of diagnostic knowledge base enables a hypothesis to be given in any form, namely, from the lower layers as a result of reasoning process or as a symptom in the respective layer. The direct disorder to every symptom whether it is in the bottom most layer or in the intermediate layer allows the fault to be concluded very precisely without waiting for the rest of the symptoms to conclude the faults in the topmost layer.

The realistic abductive reasoning model in its original form allows the reasoning mechanism to query back the user (here, the managed nodes) to confirm the missing symptoms before concluding any fault. But, since performance tuning can not be deferred for such a long time before all the required symptoms are obtained, this can be relaxed since the model allows some tolerance on the number of symptoms required to conclude reason for degradation in the network performance.

By suitably constructing the network fault knowledge model required for performance tuning, this model can be found to give very good results for the problem. A case study of Ethernet performance management, discussed in Section 5, illustrates this approach.

\section{THE ALGORITHM}

The performance management model (described as algorithm, Performance_Mgt) presented in this section accepts a set of symptoms given as soft failures from the monitoring information and identifies remedies for the set of faults concluded using Realistic_ARM. Since the knowledge base which is in the form of hyper-bipartite network is converted into a layered network, the symptoms can be allowed to enter at any stage of the inference process.

\section{Nomenclature}

1. temp_man is a set of symptoms at layer of inference. (By both, one of the covers of the previous layer and the symptoms of that layer.)

2. prim_man, is a set of symptoms available at all the layers, holds the symptoms provided by the user excluding the symptoms explored in all the previous layers (if the manifestation is present in the next layer because of dummy nodes created by Build_Layered_Net, they are retained). 
3. sec_man, is a set of symptoms available at all the layers, holds all the symptoms that are provided by the user.

4. More_Manifs, a boolean, is TRUE if there are any more symptoms found to exist at a layer by either input or when asked interactively through common disorders of the existing symptoms. Otherwise it is FALSE.

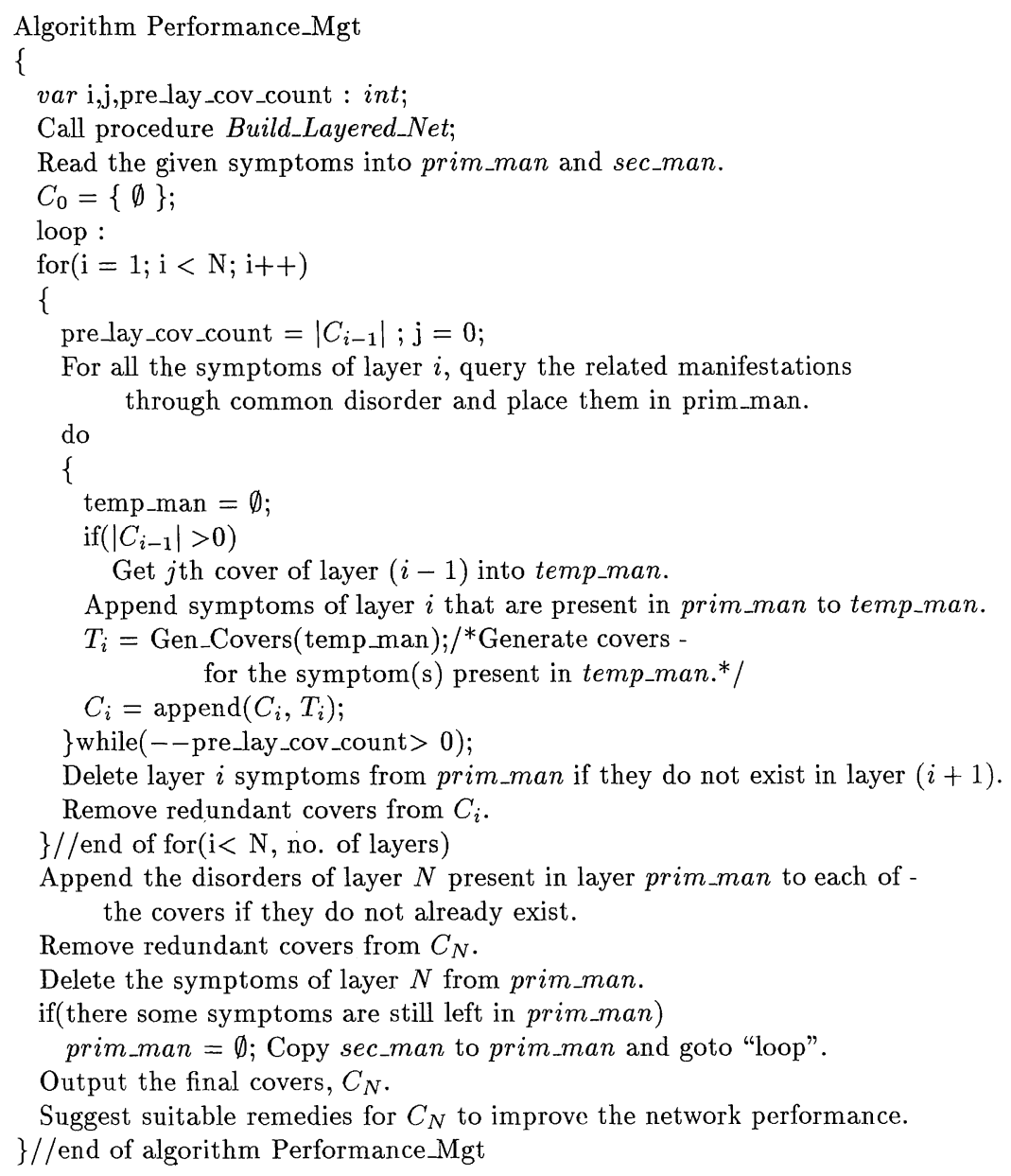




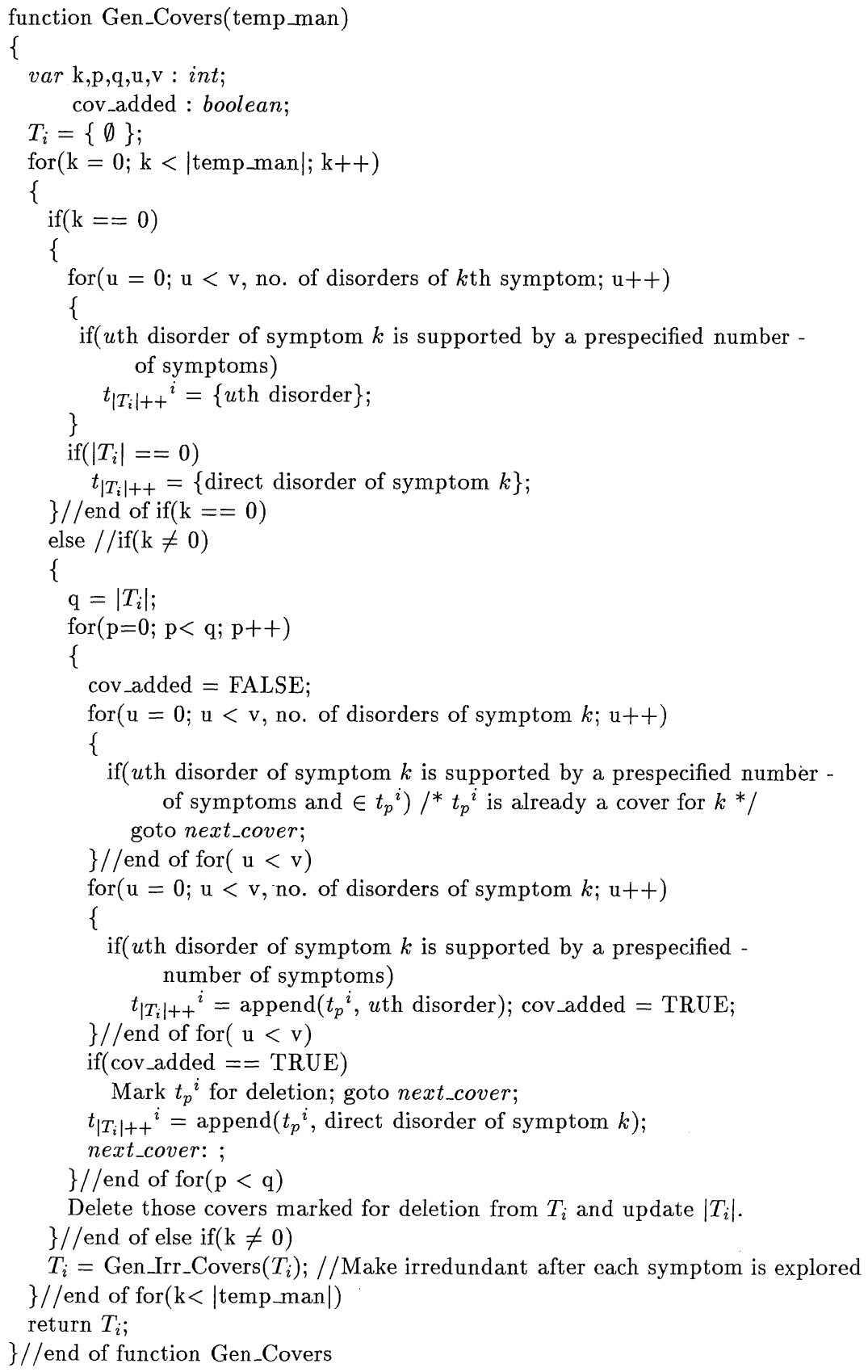


procedure Build_Layered_Net

\{

Retain the nodes of the hyper-bipartite network.

For each layer $i,(1 \leq \mathrm{i} \leq(N-2))$, of hyper-bipartite network :

if there is a link from layer $i$ to layer $(i+1)$, retain the same in the layered network.

if there is a link (say $l_{h_{m}, h_{n}}$ ) from manifestation/hypothesis at layer $i$ to hypothesis/disorder at layer $(i+k), k>1$, replace it by creating a dummy node with the name same as $h_{m}$ at all the intermediate layers and connect them.

\}//end of procedure Build_Layered_Net

\section{ETHERNET PERFORMANCE MANAGEMENT MODEL - A CASE STUDY}

In this section, we consider a restricted Ethernet model to illustrate the ideas presented in this work. We assume that reader is aware of the Ethernet operation (Metcafe, 1976), (Boggs, 1988).

We consider a Ethernet network performance management model with the following assumptions.

- The information that needs to be monitored for the purpose of performance tuning is collected from the stations and the channel. And, that information, which is beyond the normal (both above and below the normal limits) are reported as symptoms.

- Some monitoring information like load is normal and collisions are with in the range are included to support the diagnostic process by eliminating the unnecessary fault sets which otherwise raise false alarms.

- there may be some missing information and the entire information may not be available at the time of diagnosis.

\subsection{The Ethernet Performance Management Knowledge Model}

The Ethernet performance management knowledge base (Boggs, 1988), (Hansen, 1992), (Feather, 1992), (Feather, 1993) is constructed as a hyper-bipartite network (see Figure 1). This maps the network performance management knowledge onto a model suitable for the Realistic_ARM. 


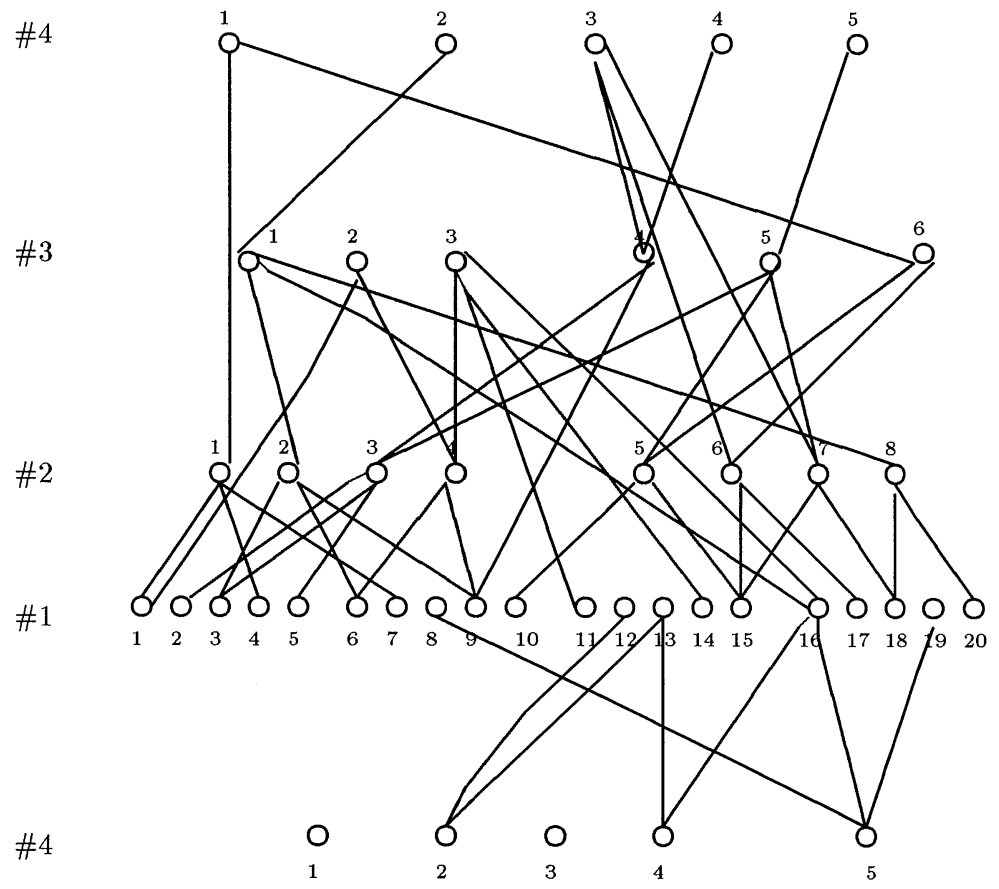

Figure 1 Ethernet Performance Management Knowledge Model. Layer 4 is shown in two places to avoid clumsiness; bottom most one connecting from layer 1 and top most one connecting from layers 2,3 .

\section{Legend:}

\section{Layer \#1:}

1. Packet loss below normal

2. Packet loss normal

3. Packet loss above normal

4. Load below normal

5. Load normal

6. Load above normal

7. Collisions below normal

8. Collisions normal

9. Collisions above normal

10.Large packets below normal

\section{Large packets normal}

12.Large packets above normal

13.Small packets below normal

14.Small packets normal

15.Small packets above normal

16. Broadcast packets normal

17.Broadcast packets above normal

18.Packet loss on spine above normal

19.Load on spine normal

20.Load on spine above normal

\section{Layer \#2 :}

1. Light traffic

2. Heavy traffic

3. Buffers are insufficient

4. Users are many
5. Preambles are many

6. Broadcast packets are many

7. Spine flooded with too many small packets

8. Heavy traffic on spine 
Layer \#3:

1. (F1) Babbling node; (Remedy, R1) : Faulty Ethernet card, report to the network manager

2. (F2) Hardware problem; (Remedy, R2) : Request the network manager to initiate Fault Diagnosis measures

3. (F3) Jabbering node; (Remedy, R3) : Ensure many packets are not above the specified size

4. Too many retransmissions

5. Under utilization of channel as many small packets are in use

6. Attempt for too many broadcasts

Layer \#4:

1. (F4) Bridge down; (Remedy, R4) : Report to the network manager

2. (F5) Network paging; (Remedy, R5) : Allocate more primary memory to the required nodes

3. (F6) Broadcast storm; (Remedy, R6) : Selectively control the broadcast packets

4. (F7) Bad tap; (Remedy, R7) : Report to the network manager along with the specified tap

5. (F8) Runt storm; (Remedy, R8) : Ensure many packets are not below the specified size

The fault knowledge base, constructed in the form of a hyper-bipartite network will be transformed into a layered network for a given diagnostic problem. The inference mechanism proceeds from the bottom most layer to the top most layer to find a solution for a given set of symptoms.

Based on a single symptom one should not conclude all its related faults which need some more symptoms to ascertain the validity. In this case, the fault corresponding to the direct disorder should be concluded. At the same time, one should be able to guess the most appropriate explanation even if a few of the symptoms are missing as is generally the case with networks due to the loss of information. Realistic_ARM can be found to solve all these problems related to the network performance management very effectively.

\subsection{Results}

The algorithm, Realistic_ARM, was run for various sets of symptoms (from Layer 1 of Figure 1) and some of the results are given in Table 1. The prespecified number of symptoms required to support any symptom before concluding a fault is set to 1 . 
Table 1 Sample results for Ethernet performance model

\begin{tabular}{|c|l|c|}
\hline Sl.No. & Symptoms & Suggested Remedy \\
\hline 1. & $3,6,12,18,20$ & $\{\mathrm{R} 5\}$ \\
2. & $1,4,10,15,17$ & $\{\mathrm{R} 4\}$ \\
3. & $3,9,18,20$ & $\{\mathrm{R} 1\}$ \\
4. & $10,15,16,18$ & $\{\mathrm{R} 8\}$ \\
\hline
\end{tabular}

From Table 1, it can be observed that the covers generated by the proposed model contain appropriate explanation for any given symptoms without much of extra guess. Otherwise, generating so many covers is computationally expensive and, further, it requires elimination of inappropriate covers using some heuristic method. The proposed model avoids these problems and still makes an appropriate guess which proves to be useful to solve the performance management problem.

To demonstrate an example, consider the soft failures given as Sl. No. 4 in Table 1. The soft failures, observed as symptoms, are number of large packets below normal (Layer \#1, 10), small packets above normal (Layer \#1, 15), packet loss on spine above normal (Layer \#1,18) and the number of broadcast packets are with in the normal range (Layer \#1,16; this is a test but not a symptom). The fault concluded is "Runt storm" and the remedy is to ensure by possible means of control that, too many small packets are not injected into the network.

\section{CONCLUSION}

The abductive reasoning has been shown to be well suited for the specialized problems of network performance management. Realistic Abductive Reasoning Model is then used to solve the network performance management problem. This approach has been illustrated with the help of Ethernet performance management model. The explanation provided by the model is appropriate and shall not have much of extra guess. The results obtained by the proposed model are more appropriate and quite encouraging.

\section{REFERENCES}

Boggs D. R., Mogul J. C., and Kent C. A. (1988) Measured Capacity of an Ethernet: Myths and Reality, Comp. Comm. Reveiw, 222-234.

Bylander T., Allenmang D., Tanner M. C., and Josephson J. R. (1991) The Computational Complexity of Abduction, Artificial Intelligence, 49, 25-60.

Cassel L. N., Patridge C. and Westcott J. (1989) Network Management Architectures and protocols : Problems and Approaches, IEEE Jl. on Selected Areas in Comm. 7(7), 1104-1114. 
Feather F. E. (1992) Fault Detection in an Ethernet Network via Anomaly Detectors, Ph.D. thesis, Dept. Electrical and Computer Engineering, Carniegie Mellon University.

Feather F. E., Slewlorek D. and Maxion R. (1993) Fault Detection in an Ethernet Network Using Anomaly Signature Matching, Comp. Comm. Reveiw, 279-288.

Hansen J. P. (1992) The Use of Multi-Dimensional Parametric Behavior of a CSMA/CD Network for Network Diagnosis, Ph.D. thesis, Dept. Electrical and Computer Engineering, Carniegie Mellon University.

Hayes S. (1993) Analyzing Network Performance Management, IEEE Comm. Magazine, $31(5), 52-59$.

Metcafe R. M. and Boggs D. R. (1976) Ethernet : Distributed Packet Switching for Local Computer Networks, Comm. of ACM, 19(7), 395-404.

Peng Y. and Reggia J. A. (1987) Diagnostic Problem-Solving with Causal Chaining, Intl. Jl. of Intelligent Systems, 2, 395-406.

Peng Y. and Reggia J. A. (1990) Abductive Inference Models for Diagnostic ProblemSolving, Springer-Verlag, New York.

Pople H. (1973) On the Mechanization of Abductive Logic, in Proc. of Intl. Joint Conf. on Artificial Intelligence, 147-152.

Prem K. G. and Venkataram P. (1994) A Realistic Model for Diagnostic Problem Solving using Abductive Reasoning Based on Parsimonious Covering Principle, in 3rd Turkish Conf. on Artificial Intelligence and Neural Networks (TAINN'94), Ankara, Turkey, 1-10.

Reggia J. A., Nau D., Wang P. and Peng Y. (1985) A Formal Model of Diagnostic Inference, Information Sciences, 37, 227-285.

Sluman C. (1989) A Tutorial on OSI Management, Comp. Networks and ISDN Systems, $17,270-278$.

Prem Kumar Gadey received his B.Tech. ( Electronics \& Communication Engineering) from Sri Venkateswara University in 1990 and M.Tech. (Artificial Intelligence \& Robotics) from University of Hyderabad in 1992. Since then he is a Ph.D. student in department of Electrical Communicaiton Engineering, Indian Institute of Science, Bangalore. His major research interests include Communication Networks, Internetworking, Distributed Computing, Expert Systems and Artificial Neural Networks. Currently he is focussing on applying Artificial Intelligence techniques to the area of Network Management. He is a student member of IEEE Communication Society.

Pallapa Venkataram received his Ph. D. degree from The University of Sheffield, England, in 1986. He is currently an Associate Professor in department of Electrical Communication Engineering, Indian Institute of Science, Bangalore, India. He worked in the areas of Distributed Databases, Communiation Protocols and AI applicaitons in Communication Networks and has published many papers in these areas. 